\title{
Manejo didáctico de los docentes del nivel de preparatoria en la prevención del abuso sexual infantil: un estudio de caso en la Unidad Educativa Comunitaria Intercultural Bilingüe «San José de Chonta Punta» (Ecuador)
}

\section{Didactic management of high school teachers in the prevention of child sexual abuse: a case study in the schools of the Chonta Punta circuit in the province of Napo- Ecuador}

\author{
YÉPEZ HERRERA, Emerson ${ }^{1}$ \\ APOLO EGUEZ, Deysi ${ }^{2}$ \\ CAIZALUISA BARROS, Noemí ${ }^{3}$ \\ VILLLAVICENCIO ALVAREZ, Víctor ${ }^{4}$
}

\begin{abstract}
Resumen
El objetivo principal de este estudio de caso fue analizar el manejo didáctico que aplican los 18 docentes del nivel de preparatoria de la Unidad Educativa rural Comunitaria Intercultural Bilingüe "San José de Chonta Punta" provincia del Napo, cantón Tena Ecuador, para prevenir el abuso sexual. Se realizó una investigación descriptiva, con enfoque cuantitativo, orientado a la recolección y análisis de datos, en la cual se evaluó el conocimiento del docente acerca del tema, su manera de actuar dentro de clases y su experiencia. El estudio aporta nuevas perspectivas para los docentes sobre la prevención del abuso sexual infantil.
\end{abstract}

Palabras claves: abuso sexual infantil, manejo didáctico, prevención, conocimiento en el tema

\begin{abstract}
The principal objective was analyze the didactic management applied by the 18 teachers of the level high school of the Bilingual Intercultural Rural Community Educational Unit "San José de Chonta Punta" Napo province, which belongs to the Tena Ecuador canton, to prevent sexual abuse. A descriptive description was made, with a quantitative approach, oriented to the collection and analysis of data, in which it was evaluated the teacher's knowledge about the subject, his experience and his way of acting in the classroom. The study provides new perspectives for teachers on the prevention of child sexual abuse.

Keywords: child sexual abuse, didactic management, prevention, knowledge on the subject.
\end{abstract}

\footnotetext{
${ }^{1}$ Universidad de las Fuerzas Armadas-ESPE | espe - Departamento de Ciencias Humanas y Sociales.

2 Universidad de las Fuerzas Armadas-ESPE | espe - Departamento de Ciencias Humanas y Sociales.

${ }^{3}$ Universidad de las Fuerzas Armadas-ESPE | espe · Departamento de Ciencias Humanas y Sociales. Email noemi-caizaluisa@hotmail.com

${ }^{4}$ Universidad de las Fuerzas Armadas-ESPE | espe · Departamento de Ciencias Humanas y Sociales
} 


\section{Introducción}

En la actualidad hablar de abuso sexual infantil es un tema que desencadena preocupación, cada vez, los datos estadísticos de su incidencia van en aumento, se han realizado varios estudios que analizan la intervención del docente como medida de prevención.

Este trabajo presenta los resultados asociados a las metodologías que utilizan los docentes del nivel de preparatoria en la prevención del abuso sexual infantil en el aula de clase.

Las investigaciones actuales en la provincia de Pichincha, sobre el abuso sexual y la violencia intrafamiliar deben ser impartidos desde las escuelas y colegios de una manera adecuada, sin tabúes, con el personal calificado (Maestros, Trabajadoras Sociales, Psicólogos) desde un enfoque claro, sin dejar de lado la importancia o el rol que juegan los padres de familia en su educación, eliminando los patrones culturales machistas. Los padres son los principales responsables quienes deben conocer, aprender e informarse para poder orientar y educar a sus hijos (Cevallos, 2016).

\section{Antecedentes}

Según la Organización Mundial de la Salud (OMS), "se calcula que 150 millones de niñas y 73 millones de niños han experimentado relaciones sexuales forzadas u otras formas de violencia sexual que implican contacto físico" (Pinheiro, 2006, p. 12). La cantidad de niñas violentadas sexualmente es aproximadamente el doble que en los niños. En "países como Tanzania, Kenia o Zimbabue 1 de cada 3 niñas y 1 de cada 6 niños ha sufrido alguna forma de abuso sexual" (Saldivar, 2014, p. 1). Los datos arrojan que en los diferentes países de África también se presencia abuso sexual hacia los infantes, lo lamentable es que la mayoría de estos casos no suelen ser denunciados.

En "países más desarrollados como en Estado Unidos, casi un $25 \%$ de las adolescentes y un $10 \%$ de los chicos han sufrido abuso sexual" (Saldivar, 2014, p. 1). Entonces se evidencia que en EE. UU se presentan más casos de abuso sexual en los adolescentes y con un porcentaje menor en los infantes. "De acuerdo con datos de la Organización para la Cooperación y Desarrollo Económicos, México ocupa el primer lugar a nivel mundial en materia de abuso sexual, violencia física y homicidio de menores de 14 años" (Regeneración, 2017, p. 1). Se menciona que México es un país que lidera las estadísticas en maltrato infantil de cualquier tipo. En la misma revista se menciona que "aproximadamente 4.5 millones de niños mexicanos son víctimas de cualquier tipo de abuso sexual y lo alarmante es que solo $2 \%$ de los casos se llegan a conocer" (Regeneración, 2017, p. 1). Es decir, la víctima de un abuso tiene miedo de contar y de denunciar así que prefiere callar, el índice de las personas que lo denuncian es bajo para la cantidad de casos reales que existen.

Redondo Figuero (2005) en España menciona que "el abuso sexual infantil es una patología muy frecuente, puesto que tiene una incidencia anual de 0,5 casos por 1.000 niños" (p.3). En México se realizó una investigación sobre "La sexualidad positiva y prevención de abuso sexual infantil" (Velázquez, Ramírez, Álvarez, \& Limón, 2015). Con el objetivo de percibir el conocimiento que poseen los niños de primer grado en cuanto a la sexualidad. Se brindó un espacio con talleres para desarrollar un programa de autoprotección en los niños, obteniendo resultados positivos (Velázquez et al., 2015).

Según el artículo de Galet y Macedo (2016) sobre abuso/violencia infantil y derecho a la imagen en el contexto europeo y español "el $20 \%$ de los niños europeos sufren con la práctica de abusos sexuales" (p. 53). Un estudio realizado en 21 estados concluyó que España es, junto con Estados Unidos, el país con el porcentaje más elevado de casos de abuso/violencia infantil. En España, el 19\% de la población adulta admite haber sufrido abusos sexuales en algún momento de su infancia. En los varones este porcentaje es del 15,2\%, y en las mujeres, del 
22,5\% (Galet \& Macedo, 2016). En Colombia, así como en el resto de Latinoamérica, el abuso sexual infantil es un problema que va en aumento, según Vélez, Henao, Ordóñez, \& Gómez (2015), quienes mencionan que “Medicina Legal realizó 22.597 evaluaciones médico-legales por presuntos delitos sexuales, 87\% de las víctimas fueron niños y adolescentes" (p. 3). Es alarmante que las estadísticas suban cuando se trata de abusos a menores siendo un grupo prioritario.

De acuerdo con la evidencia científica las mujeres tienen entre 1,5 y 4 veces más riesgo de abuso sexual infantil que los hombres, y se ha identificado que la edad de inicio del abuso mayoritariamente se ubica entre los 8 y 12 años, con un segundo pico entre los 6 y 7 años. (Vélez et al., 2015, p.5)

Estos datos generalmente son similares en los diversos países de nuestra región, debido a las cercanías y similitudes, las realidades que se viven, pero por sobre todo porque esta problemática se extiende a nivel mundial. Muchos casos se podrían prevenir si se tuviesen los conocimientos necesarios sobre estos temas. En Perú se realizó una investigación sobre el conocimiento que los cuidadores de niños tienen sobre violencia familiar, en donde "el 62 \% de los encuestados obtuvo un nivel de conocimiento bajo en violencia física por negligencia; el $52 \%$, en violencia sexual, y el 34 \%, en violencia psicológica." (Chanamé Ampuero, et al., 2016, p. $30)$, concluyendo que la instrucción de padres o cuidadores de menores es deficiente en lo que concierne a temas de prevención de abuso sexual infantil.

En Ecuador, el fenómeno del abuso sexual infantil, no es diferente al resto de Latinoamérica, en los medios de comunicación del país salieron a flote reveladoras noticias sobre abusos infantiles que en la mayoría de los casos se suscitaron dentro del contexto escolar, tanto como en el familiar. Según el diario El Comercio se presentaron 2673 denuncias de violencia sexual, entre enero del 2014 y el 22 de marzo del 2018, el 57\% es decir, 1524 casos corresponden a casos ocurridos dentro de las instituciones educativas según el Ministerio de Educación. Según el canal de noticias digital e internacional Vice News, hasta noviembre de 2016 no había ninguna campaña en las instituciones sobre la prevención del abuso sexual infantil en el Ecuador (Grande \& Brunner, 2018). El diario El Universo presentó, "en 2018 se registra un aumento del 55\% de las denuncias sobre casos de violencia sexual a menores en instituciones educativas. La Comisión señaló que hay 734 casos judicializados" (p. 1). En los diferentes medios informativos del país se habla de numerosas cifras de abusos sexuales contra los niños, entre los hechos más expuestos en las noticias está el caso en Quito de un profesor que abusó de sus 41 alumnos en el colegio AAMPETRA, los dos casos en Cuenca sobre el abuso a menores por parte de los sacerdotes, o los casos revelados en Guayaquil dentro de las instituciones educativas por parte de los profesores. Debido a los diferentes casos presentados, la Asamblea Nacional del Ecuador creó una comisión ocasional para investigar casos de abuso sexual, la cual como su nombre lo indica, "se encarga de investigar casos de abuso sexual a niños, niñas y adolescentes en escuelas y colegios del país" (Asamblea Nacional, 2017, p. 1). El nombre de esta comisión es Aampetra llamado así en mención al colegio donde se suscitó el abuso a todos los niños del salón por parte del profesor (Noticias Asamblea Nacional, 2017).

Como se mencionó anteriormente se ha evidenciado muchos casos de abuso sexual infantil. El Instituto Nacional de Estadísticas y Censos, indica que 1 de cada 4 mujeres han sido abusadas sexualmente (INEC, 2011). Según la Dirección de Política Criminal de la Fiscalía General del estado, en Ecuador, las tres primeras provincias donde se indican altos índices de abuso sexual son: Pichincha con el 22,77\%, Guayas con el 19,33\% y Manabí con 8,17\%, estas cifras fueron obtenidas de enero a noviembre de 2017. Según la Fiscalía General del Estado en el 2016, "la tasa de denuncias en delitos sexuales por cada 100.000 habitantes de niños, niñas y adolescentes alcanza 64 en Zamora Chinchipe, 56 en Sucumbíos, 116 en Santo Domingo de los Tsáchilas, 130 en El Oro y 181 en Azuay" (Fiscalía, 2017, p. 1). Es evidente que El Oro y Azuay son las provincias con un índice más alto. Las víctimas de abuso sexual son en un $90 \%$ niñas y en un $10 \%$ niños (Fiscalía, 2017). 
Sobre las consecuencias del abuso sexual y las repercusiones psíquicas en esta población afectada se habla en la teoría de Desarrollo Psicosexual de Freud (1976), caracterizada por elaborar la teoría del trauma que se centra en la sexualidad y se fija en los daños psicológicos. Tackett y Finkelhor (1993) mencionan los efectos emocionales que evidencian, así como los "cognitivos y sociales que se relacionan con la experiencia de abuso sexual" (Nevado, 2008, p.135), para profundizar más Trickett y McBride-Chang (1995), detallan impacto psicológico del maltrato infantil, además explican las consecuencias psicológicas del abuso sexual (Pereda, 2009). Para investigar la temática se obtuvo información de un documento elaborado por la Secretaria de Educación Pública de México (2016), llamado "Orientaciones para la prevención, detección y actuación en casos de abuso sexual infantil, acoso escolar y maltrato en las escuelas de educación básica", el cual sirvió de ayuda para verificar algunas pautas sobre cómo actuar ante los posibles casos de abuso sexual infantil, así también como en la revista Papeles del Psicólogo en España que publicó un artículo sobre "Consecuencias psicológicas iniciales del abuso sexual infantil" por Pereda Beltrán,(2009).

El abuso sexual infantil es un problema social en el Ecuador, que ha generado preocupación e interés al Estado, Ministerio de Educación y padres de familia. Los docentes de la Unidad Educativa Comunitaria Intercultural Bilingüe "San José de Chonta Punta" tienen escaso conocimiento sobre la prevención del abuso sexual infantil, esto ha provocado una falta de competencia en el abordaje de esta problemática en el aula. El detonante suele ser el bajo conocimiento sobre el manejo didáctico, estrategias para su prevención y ausencia de herramientas pedagógicas; en conjunto pueden ocasionar en el niño daños psicológicos y problemas de aprendizaje, que se ven reflejados en la clase, con carencia de habilidades personales y sociales, falta de comunicación y barreras para el aprendizaje, (Ministerio de Educación del Ecuador y la Subsecretaría para la Innovación Educativa y el Buen Vivir, 2016). Así también en la esfera psicológica se evidencia en los niños baja autoestima, aislamiento escolar, conductas auto lesivas o suicidas, incapacidad de confiar en personas adultas, presentan comportamientos compulsivos y problemas psicosomáticos, acompañados de un bajo rendimiento estudiantil y hasta el último de los casos la deserción escolar (Cantón-Cortes \& Cortes, 2015).

\section{Marco de Referencia}

\subsection{Definición del Abuso Sexual Infantil}

El abuso sexual infantil es una problemática a nivel mundial que se ha venido desarrollando desde tiempos pasados en diferentes clases sociales. "El abuso sexual ocurre cuando un niño es utilizado para la estimulación sexual de su agresor o la gratificación de un observador" (UNICEF, 2016, p. 7). Es decir, una persona mayor en edad a la víctima utiliza al infante para su satisfacción sexual.

La persona que abusa del menor por lo general es alguien que se encuentra en su entorno familiar o escolar, aunque tampoco se descarta un desconocido. El ASI implica toda interacción sexual con el infante con y sin su consentimiento que, mediante engaños, amenazas, manipulación o soborno se utiliza para acercarse al niño y lograr su objetivo, puede ser con tocamiento y sin tocamiento. Cualquier persona que sobrepase la edad del infante y tenga un contacto sexual con el menor es considerado como abusador.

Arredondo (2002) menciona que el abuso sexual en el infante puede o no incluir tocamiento genital o de otras partes del cuerpo, el agresor muestra sus órganos sexuales, obliga al niño a tocarlos, también es cualquier tipo de penetración o intento de ella con alguna parte del cuerpo o con objetos, realizar o enseñar pornografía al niño o la niña, todo esto puede ocurrir en un mismo momento o por separado, en una sola ocasión o durante un período prolongado de tiempo (Velázquez et al., 2015).

El abuso sexual infantil es una forma de maltrato infantil en la que se produce daños físicos y psicológicos. Es la peor forma en la que se irrespeta a un menor irrumpiendo sus derechos. 


\subsection{Consecuencias del Abuso Sexual Infantil}

Según Peroni y Prato (2012), el impacto del abuso sexual infantil suele ser muy variado, el trauma puede ser muy notable teniendo afectaciones psicológicas, físicas, conductuales, emocionales y sociales, mientras que en otros casos las secuelas no son tan perceptivas, se habla de una experiencia negativa, pero con consecuencias mínimas. No todos los niños manifiestan el mismo grado de afectación, para cada niño las consecuencias son diferentes esto dependerá de distintos factores como:

- Tipo de violencia sufrida

- Tiempo de duración de la agresión

- Quién es el agresor

- Características individuales del niño, la niña o el adolescente

- Edad del niño, la niña

- Entorno y resiliencias (individual y familiar)

- Reacción del entorno (familia) frente a la agresión

Las afectaciones de un trauma como el Abuso Sexual Infantil se podrán evidenciar a corto y largo plazo

\subsection{La Prevención Del Abuso Sexual Infantil}

"Prevenir supone reducir los factores de riesgo y aumentar los factores de protección" (Villanueva, 2005, p.21). Es decir, hay que anticiparse ante cualquier hecho que pueda ocurrir, por eso prevenir es cualquier acción que impide la aparición de un problema y desencadenamiento de consecuencias negativas.

Lo que se quiere es estar preparados para un evento de este tipo, que puede estar en nuestra realidad como docentes. Cuando una persona está preparada, por consecuencia tendrá un alto conocimiento sobre cómo actuar en una situación de la manera adecuada.

Hay que tener en cuenta que un caso de abuso sexual infantil puede aparecer en cualquier instante y es oportuno saber actuar, por eso el objetivo de este trabajo es saber cuál es el rol que un docente debe cumplir al enterarse de un caso de abuso sexual, qué hacer para prevenir, cuáles son las señales de alarma que un niño emana después de trauma ASI, debe saber cómo enseñar a proteger a los niños con temas sobre el cuidado de su cuerpo, tener en claro el proceso para reportar un caso de abuso sexual.

\subsubsection{Protocolo de acción ante casos de violencia sexual en el Ecuador}

El Ministerio de Educación del Ecuador y la Subsecretaría para la Innovación Educativa y el Buen Vivir, (2016). Presenta el siguiente protocolo de actuación ante casos de violencia sexual que debe ser aplicado de forma obligatoria en todos los establecimientos de educación fiscal, fisco misional, municipal y particular.

El primer paso es la detección, si el/la docente sospecha o está seguro de que un alumno está siendo o fue abusado sexualmente debe informar a las autoridades y al Departamento de Consejería Estudiantil en el caso de que no se sospeche que ellos sean los agresores. El personal del DECE son los delegados para abordar estos problemas (Ministerio de Educación Ecuador, 2017).

La intervención es el segundo paso donde el Departamento de Consejería Estudiantil, es el encargado de realizar el informe de violencia o abuso en el niño. Este departamento se encarga de comunicar a los representantes legales (siempre y cuando no sean los presuntos abusadores) y comentar sobre el informe y las medidas que tomará la institución educativa. La situación podría ser reportada por la víctima o por una tercera persona, es 
importante no realizar preguntas a las víctimas más de lo que ella quiere comentar, para evitar una revictimización. La prioridad de la institución debe ser preservar el bienestar del niño, además de llevar a cabo un código de confidencialidad y no exponerlo, (Ministerio de Educación Ecuador, 2017).

La derivación es el tercer paso, el Departamento de Consejería Estudiantil o las autoridades deben precautelar la salud del infante, en el caso de emergencia médica se le debe acompañar a un establecimiento médico o llamar al ECU911. Con el informe por parte del Departamento de Consejería Estudiantil se debe realizar una denuncia en máximo 24 horas ante la Fiscalía y poner en conocimiento a la dirección distrital de Educación. Las Unidades Judiciales y la Junta Cantonal de protección de Derechos coordinan acciones para garantizar la protección de niños, niñas y adolescentes (Ministerio de Educación Ecuador, 2017).

Las personas que trabajan en el DECE deben abrir un espacio en el cual se brinde apoyo emocional y tratamientos psicológicos a la víctima, esto dependerá de la gravedad de la situación.

El seguimiento es el cuarto paso, las autoridades de la institución educativa deben garantizar el cumplimiento de las medidas de protección y colaborar en el proceso de investigación. El DECE debe realizar el seguimiento y el acompañamiento oportuno en el proceso del infante, deben estar pendiente del desarrollo académico, físico, emocional y social del niño dentro y fuera del plantel educativo (Ministerio de Educación Ecuador, 2017).

Los docentes deben trabajar como apoyo al DECE en todas las acciones a tomar con el infante, ayudando con el cumplimento de sus tareas y otras responsabilidades del niño.

\subsubsection{El currículo: contenido para la prevención de abuso sexual infantil}

En el Ecuador dentro de los Ejes de Desarrollo y Aprendizaje del currículo de Educación General Básica Preparatoria (2016), se encuentra el de Desarrollo Personal y Social en el que el ámbito de identidad y autonomía, hace hincapié en temas específicos que deben enseñar los docentes a los niños, como las partes del cuerpo y su funcionamiento. Además deben formar a los menores en reconocer lo que sienten y piensan y estimularlos para que sean expresivos, con el fin de que si se suscita un abuso él tenga la confianza para hablar y contar lo que le sucede.

En el ámbito de convivencia se habla de enseñar al niño sobre los derechos y responsabilidades que tienen (Ministerio de Educación, 2016), otro tema importante por ver es que los niños aprendan que cada persona tiene diferencias individuales físicas y de carácter, entre compañeros y las demás personas. En el ámbito de expresión corporal debemos abarca el reconocimiento de los estados de ánimo, sensaciones, emociones, se debe hacer énfasis en que existen sensaciones agradables y desagradables, diferenciar que cuando algo nos haga sentir incómodo debemos contarlo a una persona de nuestra confianza, eso es parte de expresar nuestros sentimientos, ya que es necesario que las demás personas sepan cómo nos sentimos para poder ayudar.

En base a lo visualizado en el currículo de educación de preparatoria se rescata varios ámbitos en los que se presentan temas de cuidado en el niño y se puede direccionar a temas de prevención, pero el problema es que no muchos de los docentes optan por enfocarse en temas para la prevención de abusos sexuales sino en cumplir los contenidos sin darles mucha importancia. Además, el currículo no se presta para hablar exclusivamente sobre temas de prevención en el abuso sexual infantil, no hay temas sobre la autoprotección, o del cuidado del cuerpo enfocado en que nadie debería tocarlos, no hay un espacio que hable lo que es un abuso, y peor aún en ninguna parte del currículo se enseña lo que debe hacer una docente en caso de sospechar un abuso sexual infantil.

\section{Metodología}

El presente estudio se efectuó mediante una investigación de campo con enfoque cuantitativo, se aplicó un cuestionario que consta de 12 preguntas, 10 tipo escala de Likert y dos dicotómicas, el mismo que fue revisado 
y sometido a validación con un alfa de Cronbach de 0,842. La aplicación del instrumento se realizó de manera presencial a los docentes en la provincia de Napo- Ecuador ciudad del Tena, en la escuela Chonta Punta.

El estudio fue estructurado en cuatro etapas: La primera, denominada planificación y diseño de documentos, en la segunda etapa, se aplicó un cuestionario dirigido a un total de 18 docentes del nivel de preparatoria de la escuela Chonta Punta.

Tras la aplicación de los instrumentos y recogida de los datos, se ejecutó la tercera etapa, la cual permitió examinar la información obtenida. Las respuestas del cuestionario fueron evaluadas mediante el programa de análisis de datos SPSS (versión 22).

La última etapa se verá reflejada en los resultados obtenidos del análisis estadístico.

\section{Resultados}

El siguiente apartado describe las respuestas recopiladas de los docentes de la escuela Chonta Punta respecto al abuso sexual infantil en sus distintas dimensiones que se ven refleja en la figura 1.

Figura 1

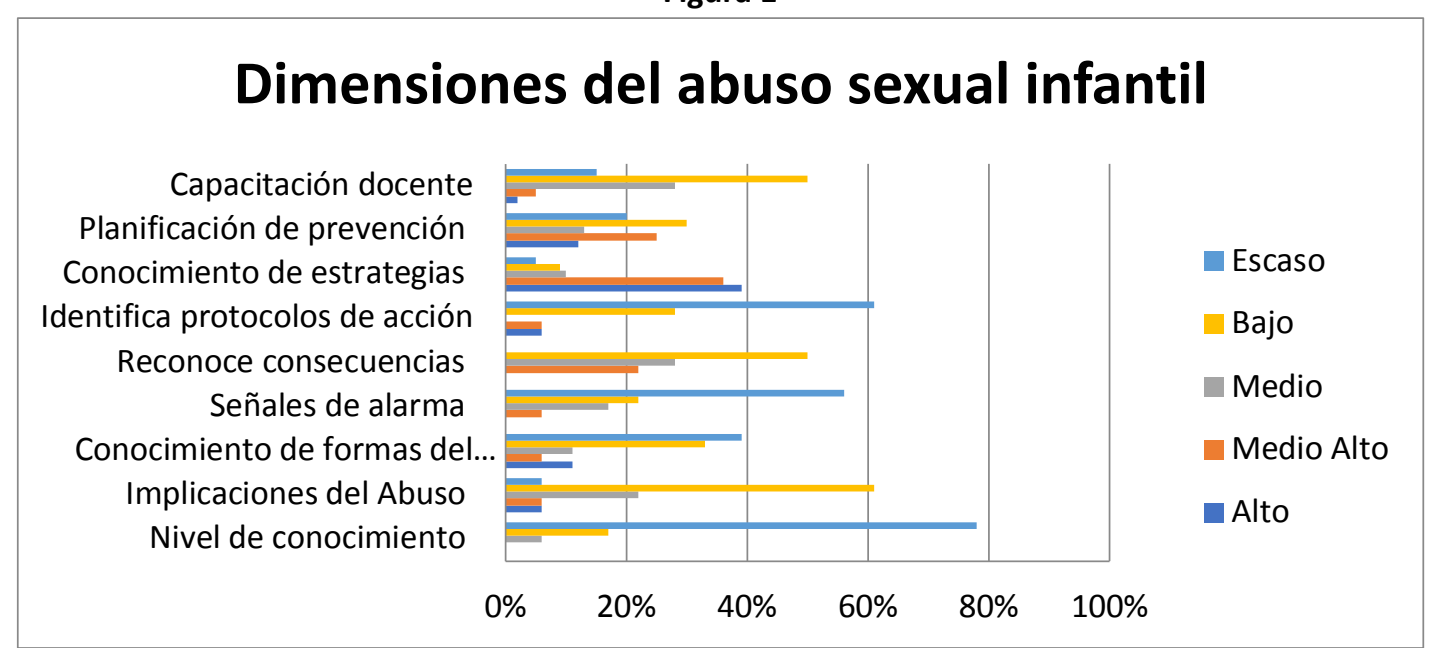

La información fue recolectada por medio de un cuestionario, elaborado ad hoc. Esta sección abarca ítems de respuestas cerradas por medio de una escala tipo Likert con valores que van de 1 a 5 , donde 1 significa "alto"; 2 , "medio alto"; 3, "medio"; 4, "bajo" y 5, "escaso", las preguntas abarcan las siguientes dimensiones referentes al abuso sexual infantil como: implicaciones del abuso sexual infantil, formas de abuso, señales de alarma de abuso, consecuencias del abuso sexual infantil, protocolo de acción y capacitación docente. Además, de dos preguntas dicotómicas orientadas a las siguientes dimensiones: conocimiento-estrategias de abuso sexual infantil y planificación de prevención del abuso sexual infantil.

Los resultados de las dimensiones del abuso sexual infantil presentados en la figura 1 se detallan de la siguiente manera: dimensión 1 (Nivel de conocimiento) evidencia que un $78 \%$ de docentes poseen un escaso conocimiento, bajo 17\%, medio 6\%;dimensión 2 (Implicaciones del Abuso) evidencia que es 6\% escaso, bajo $61 \%$, medio $22 \%$, medio alto6\%, alto $6 \%$; dimensión 3 (Conocimiento de formas del Abuso Sexual Infantil) escaso $39 \%$, bajo 33\%, medio 11\%, medio alto 6\%, alto 11\%; dimensión 4 (Señales de alarma) escaso 56\%, bajo 22\%, medio $17 \%$, medio alto 6\%;dimensión 5 (Reconoce consecuencias) bajo $50 \%$, medio $28 \%$, medio alto 22\%; dimensión 6 (Identifica protocolos de acción) escaso 61\%, bajo 28\%, medio alto 6\%, alto 6\%; dimensión 7 (Conocimiento de estrategias) escaso $5 \%$, bajo $9 \%$, medio $10 \%$, medio alto $36 \%$, alto $39 \%$, dimensión 8 
(Planificación de prevención) escaso 20\%, bajo 30\%, medio 13\%, medio alto 25\%, alto 12\%; dimensión 9 (Capacitación docente) escaso $15 \%$, bajo $50 \%$, medio $28 \%$, medio alto $5 \%$, alto $2 \%$

\section{Conclusiones}

Los docentes del nivel de preparatoria del Circuito de Chonta Punta presentan un conocimiento medio alto sobre las consecuencias, formas de abuso, estrategias para prevenir el abuso sexual infantil.

Necesitan mayor capacitación sobre protocolos de acción y manejo didáctico para tratar temas de prevención del abuso sexual infantil dentro del aula de clase.

A pesar de las campañas comunicacionales de prevención del Ministerio de Educación con Unicef: 'Aprende a ser tu propio Superhéroe' y 'Conviértete en un súper padre para combatir el abuso sexual', todavía se requiere de un trabajo logístico que abarque todas las provincias y sectores del Ecuador.

El Instituto Nacional de Estadísticas y Censos, indica que 1 de cada 4 mujeres han sido abusadas sexualmente (INEC, 2011). Según la Dirección de Política Criminal de la Fiscalía General del estado, en Ecuador, las tres primeras provincias donde se indican altos índices de abuso sexual son: Pichincha con el 22,77\%, Guayas con el $19,33 \%$ y Manabí con $8,17 \%$, estas cifras fueron obtenidas de enero a noviembre de 2017. Según la Fiscalía General del Estado en el 2016, "la tasa de denuncias en delitos sexuales por cada 100.000 habitantes de niños, niñas y adolescentes alcanza 64 en Zamora Chinchipe, 56 en Sucumbíos, 116 en Santo Domingo de los Tsáchilas, 130 en El Oro y 181 en Azuay" (Fiscalía, 2017, p. 1). Es evidente que El Oro y Azuay son las provincias con un índice más alto. Las víctimas de abuso sexual son en un 90\% niñas y en un 10\% niños (Fiscalía, 2017).

\section{Referencias Bibliográficas}

American Academy of Pediatrics. (2016). healthy children.org. Obtenido de https://www.healthychildren.org/Spanish/ages-stages/preschool/Paginas/sexual-behaviors-youngchildren.aspx

Berlinerblau, V. (noviembre de 2016). Abuso sexual contra niños, niñas y adolescentes: Una guía para tomar acciones y proteger. Obtenido de Fondo de las Naciones Unidas para la infancia (UNICEF): https://www.unicef.org/ecuador/proteccion-AbusoSexual_contra_NNyA-2016_\%281\%29.pdf

Cantón-Cortes, D., \& Cortes, M. R. (2015). Consecuencias del abuso sexual infantil: una revisión de las variables intervinientes. Anales de Psicología, 552-561.

Cantón-Cortes, D., \& Cortes, M. R. (2015). Consecuencias del abuso sexual infantil: una revisión de las variables intervinientes. Anales de Psicología, 552-561. Obtenido de http://scielo.isciii.es/scielo.php?script=sci_arttext\&pid=S0212-97282015000200024

Cevallos, D. M. (2016). El abuso sexual y la violencia intrafamiliar como factores para el ingreso de menores de edad al sistema de acogimiento institucional en la Mini Aldea Hogar Suizo, primer semestre del año 2016. Obtenido de http://www.dspace.uce.edu.ec/handle/25000/13027

Chanamé Ampuero, E., Danjoy León, D. L., Cerna, C., Zurita, J., Valentín, R., \& Roque, M. (2016). Conocimientos sobre violencia familiar en padres o cuidadores de escolares de un colegio de educación básica. Red de Revistas Científicas de América Latina y el Caribe, España y Portugal, 29-40.

CONGRESO NACIONAL. (03 de enero de 2003). Código de la Niñez y Adolescencia. Obtenido de http://unl.edu.ec/sites/default/files/contenido/transparencia/codigo_ninez.pdf 
Darriulat, N. E. (Julio de 2017). Abuso Sexual Infantil: sus consecuencias a corto y largo plazo. Obtenido de https://sifp.psico.edu.uy/sites/default/files/Trabajos\%20finales/\%20Archivos/trabajo_final_de_grado._darriulat_n._2017.p df

Echeburúa, E., \& Corral, P. d. (2006). Emotional consequences in victims of sexual abuse in childhood. . Obtenido de http://scielo.isciii.es/scielo.php?pid=\$1135-76062006000100006\&script=sci_arttext\&tIng=en

Educación, M. d. (2018). Guía básica para prevenir y actuar frente a la violencia sexual en el sistema educativo. Obtenido de https://educacion.gob.ec/wp-content/uploads/downloads/2018/10/Guia-Super-Profes.pdf

Galet, \& Macedo. (2016). Abuso/violencia infantil y Derecho a la Imagen en el contexto europeo y español. Obtenido de http://emaberto.inep.gov.br/index.php/emaberto/article/view/2734/2567

Grande, R., \& Brunner, E. (30 de 04 de 2018). La historia de abuso sexual en escuelas que estremeció a Ecuador. Vice News. Obtenido de https://www.vice.com/es_latam/article/3k7yjv/vice-news-la-historia-de-abusosexual-en-escuelas-que-estremecio-ecuador

Heredia Carrión, J. V. (2018). INTERVENCIÓN EN CRISIS DE ACUERDO A LOS PRIMEROS AUXILIOS PSICOLÓGICOS. Obtenido de http://repositorio.utmachala.edu.ec/bitstream/48000/12793/1/ECUACS-2018-PSCDE00031.pdf

Hernández Sampieri, R., Fernández, C., \& Baptista, M. (2014). Metodología de la investigación. México D.F: McGRAW-HILL EDUCATION.

Medina, Kahn, Huerta, Sánchez, Calixto, Sánchez; Vega. (2015). Neurodesarrollo infantil: características normales y signos de alarma en el niño menor de cinco años. Obtenido de https://rpmesp.ins.gob.pe/index.php/rpmesp/article/view/1693/1776

Ministerio de Educación del Ecuador y la Subsecretaría para la Innovación Educativa y el Buen Vivir. (2016). Orientaciones para la prevención, detección y actuación en casos de abuso sexual infantil, acoso escolar y maltrato en las escuelas de educación básica. Obtenido de https://www.gob.mx/cms/uploads/docs/Orientaciones_211216.pdf

Ministerio de Educación Ecuador. (2017). Protocolos de actuación frente a situaciones de violencia detectadas o cometidas en el sistema educativo. Ecuador. Obtenido de https://educacion.gob.ec/wpcontent/uploads/downloads/2017/03/Protocolos_violencia_web.pdf

Ministerio de Educación. (2016). Currículo de Educación General Básica Preparatoria. Obtenido de https://educacion.gob.ec/wp-content/uploads/downloads/2016/03/PREPATORIO.pdf

Ministerio de salud pública y asistencia social. (2006). Modelo de intervención en crisis en situaciones de emergencias y desastres. El Salvador, El Salvador. Obtenido de http://asp.salud.gob.sv/regulacion/pdf/otrosdoc/modelo_intervencion_situaciones_emergencias.pdf

Mortimer, V. (2018). Mitos y verdades sobre el abuso sexual infantil. La Nación. Obtenido de https://www.lanacion.com.ar/2125398-mitos-y-verdades-sobre-el-abuso-sexual-infantil

NCTSN, R. N. (febrero de 2013). Folleto Informativo Sobre el Abuso Sexual Infantil. Obtenido de https://www.nctsn.org/sites/default/files/resources//child_sexual_abuse_fact_sheet_parents_teachers_caregivers_sp.pdf

Peroni, G., \& Prato, J. (Abril de 2012). Aportes para la intervención en maltrato y abuso sexual infantil y adolescente. Obtenido de http://www.buentrato.org.uy/wp-content/uploads/2013/10/Aportes-abuso13-412FINALWeb.pdf 
Pinheiro, P. S. (2006). INFORME MUNDIAL SOBRE LA VIOLENCIA CONTRA LOS NIÑOS Y NIÑAS. Obtenido de https://www.unicef.org/mexico/spanish/Informe_Mundial_Sobre_Violencia.pdf

Redondo Figuero, C. (2005). El abuso sexual infantil. Boletín de la sociedad de pediatría de Asturias, Cantabria, Castilla y León, 3.

Regeneración. (2017). México primer lugar a nivel mundial en abuso sexual a menores: OCDE. Obtenido de https://regeneracion.mx/mexico-primer-lugar-a-nivel-mundial-en-abuso-sexual-a-menores-ocde/

Salas, L. (s,f). Intervención en crisis en el abuso sexual. Obtenido de http://www.binasss.sa.cr/revistas/ts/v24n551999/art1.pdf

Saldivar, E. (2014). UNICEF: la violencia infantil en cifras. Obtenido de http://noticias.universia.es/actualidad/noticia/2014/10/22/1113605/unicef-violencia-infantil-cifras.html

Save the Children. (Noviembre de 2001). Abuso sexual infantil: Manual de formación para profesionales. Obtenido de https://www.savethechildren.es/sites/default/files/imce/docs/manual_abuso_sexual.pdf

Velázquez, V., Ramírez, M., Álvarez, M., \& Limón, I. (2015). Sexualidad Positiva y prevención de abuso sexual infantil en la frontera norte de Mexico. Revista Latinoamericana de Ciencia Psicológica, 7,9-16. Obtenido de http://www.psiencia.org/ojs/index.php/psiencia/article/view/136/180

Vélez, C. M., Henao, P. A., Ordóñez, F., \& Gómez, L. E. (2015). Evaluación de un programa de promoción de conductas de Autoprotección para la prevención del abuso sexual infantil, Medellín, Colombia . Revista Médica de Risaralda, 3-8.

Villanueva, I. (septiembre de 2013). El abuso sexual infantil: Perfil del abusador, la familia, el niño víctima y consecuencias psíquicas del abuso. Obtenido de http://oaji.net/articles/2017/1787-1485548200.pdf

Viviano, T. M. (2012). Abuso sexual: Estadísticas para la reflexión y pautas para la prevención. Obtenido de https://www.mimp.gob.pe/files/programas_nacionales/pncvfs/libro_abusosexual.pdf

Esta obra está bajo una Licencia Creative Commons

Attribución-NoCommercial 4.0 International

\section{(c) EYY-NC}

